\title{
Original
}

\section{Experiencia inicial de nefrectomia derecha de donante vivo por laparoscopia}

\author{
Robert J. de Andrade Andrade, David S. Arana Castro, David E. Arana García, Juan C, Córdova Melo \\ Unidad de Trasplante, Hospital Militar Dr Carlos Arvelo, Caracas, Venezuela.
}

\section{Resumen}

La nefrectomía de donante vivo por laparoscopia es actualmente la técnica estándar, sin embargo, el lado derecho es controversial debido a la longitud corta de la vena renal y la incidencia de trombosis venosa.

Métodos: Se realizo un estudio prospectivo de los pacientes donantes vivos desde mayo del 2006 al septiembre 2008 en las cuales se le realizó nefrectomía derecha de donante vivo por laparoscópica. La colocación de trocares fue de forma habitual y el abordaje transperitoneal. Se utilizó la incisión de Gibson para la extracción.

Resultados: De los 10 pacientes seleccionados, se excluyó 1 debido a conversión a técnica abierta. Los criterios de lateralidad fueron sexo, volumen renal y anatomía vascular compleja. 6 pacientes ameritaron cirugía reconstructiva con prótesis vascular debido a la longitud de la vena renal. El tiempo quirúrgico promedio fue de 158.3 minutos y el sangrado promedio 272 cc. Tiempo de isquemia caliente promedio fue 3.2 minutos. La hospitalización promedio fue de 1.6 días. 1 receptor presentó función retardada de injerto.

Conclusiones: La nefrectomía derecha de donante vivo por laparoscopia ofrece una excelente calidad de injerto, siendo una técnica factible y segura, manteniendo el principio de dejar el mejor riñón al donante.

Palabras claves: Nefrectomía derecha de donante. Laparoscopia

\section{Experience initial right side laparoscopic live donor}

\section{Abstract}

Live donor nephrectomy laparoscopic technique is now standard. However, the right side is controversial because of the short length of the renal vein and the incidence of venous thrombosis.

Methods: A prospective study of patients live donors since May 2006 to September 2008 in which right nephrectomy was performed by laparoscopic live donor. The placement of trocares was usual and the transperitoneal approach. Incision was used for the extraction of Gibson.

Results: Of the 10 selected patients, 1 was excluded due to conversion to open technique. The criteria for lateralization were sex, renal volume and complex vascular anatomy. 6 patients had made back-table reconstruction surgery with prosthetic vascular due to the length of the renal vein. The average operative time was 158.3 minutes and the bleeding averaged $272 \mathrm{cc}$. Warm ischemia time averaged 3.2 minutes. The average hospital stay was 1.6 days. 1 recipient presenting delayed graft dysfunction

Conclusions: Laparoscopic live donor right nephrectomy offers an excellent quality of graft, being a technique feasible and safe, maintaining the principle of leaving the best kidney donor.

Keywords: Right donor nephrectomy. Laparoscopy.

$\mathrm{E}$ 1 trasplante renal es el tratamiento ideal para la falla renal, ya que representa un impacto tanto en la sobrevida y calidad de vida para el paciente ${ }^{1}$. Desafortunadamente, la oferta de órganos de cadáver es menor a la demanda, por lo que la nefrectomía de donante vivo ha surgido como una alternativa ante este problema, disminuyendo el tiempo de espera para el trasplante y ofreciendo una sobrevida del injerto renal superior al ser comparado con el trasplante renal de cadáver ${ }^{2}$.

La nefrectomía de donante por vía laparoscópica fue realizada en modelo animal por Gill en
$1994^{3}$ y un año más tarde, Ratner ${ }^{4}$ describe la primera nefrectomía de donante vivo en humanos, ganando aceptación en forma progresiva. En un principio el lado que se realizaba era el izquierdo debido a la longitud de la vena renal ${ }^{5}$, mientras que el lado derecho se reporta en el 2001 mostrando una alta incidencia de trombosis venosa y pérdida del injerto renal ${ }^{6}$. Las indicaciones más frecuentemente para la nefrectomía derecha son múltiples arterias o venas izquierdas, riñón derecho pequeño o con presencia de quistes $^{7,8}$. 
El objetivo de este artículo es describir nuestra experiencia en la nefrectomía derecha de donante vivo por laparoscopia.

\section{MATERIALES Y MÉTODOS}

Se realizó un estudio descriptivo de la nefrectomía derecha de donante por laparoscopia en el período comprendido desde mayo 2006 hasta septiembre 2008, realizada en la Unidad de Trasplante del Hospital Militar Dr Carlos Arvelo. Los potenciales donantes fueron evaluados, incluyendo la historia médica, examen físico, pruebas de función renal y la tomografía helicoidal con reconstrucción 3D para determinar el volumen renal y la anatomía vascular.

Los criterios de selección del riñón derecho se basaron en volumetría renal, sexo, anatomía vascular o anomalía renal izquierda.

La cirugía fue realizada bajo anestesia general inhalatoria y el paciente colocado en decúbito lateral izquierdo teniendo en cuenta los puntos de apoyo a fin de evitar lesiones por compresión. Se colocó sonda de Foley para cuantificar diuresis y nasogástrica a fin de descomprimir el estómago. La cavidad abdominal fue insuflada con dióxido de carbono hasta llegar a $12 \mathrm{mmHg}$ de presión, y se colocaron 4 trócares, en posición habitual para la nefrectomía. Inicialmente se realizó incisión en la línea de Told y maniobra de Kocher de manera de exponer la superficie anterior de la fascia de Gerota y el retroperitoneo. El uréter es identificado y disecado con suficiente tejido periureteral para evitar su desvascularización, hasta llegar al cruce de los vasos ilíacos primitivos, donde es seccionado previa colocación de Hem-o-lock ${ }^{\circledR}$ (Weck Closure Systems, Research Triangle Park, NC, EUA). La arteria y la vena renal fueron disecadas hasta su origen, evitando la elongación de la arteria. El riñón es disecado, separando la glándula adrenal del polo superior del riñón. El lugar de la extracción se realiza a través de una incisión de Gibson, dejando indemne el peritoneo. La arteria y luego la vena renal es seccionada posterior a la colocación de 2 Hem-o-lock ${ }^{\circledR}$ (Weck Closure Systems, Research Triangle Park, NC, EUA), se abre el peritoneo y se extrae el riñón en forma manual, para dar inicio a la perfusión con solución de preservación fría. La hemostasia es verificada y la sintesis de las incisiones es realizada.

En el postoperatorio, el paciente inicia su vía oral a las 6 horas previa evaluación. El manejo del dolor fue con Meperidina y el retiro de la sonda uretrovesical a las 24 horas.
El tiempo de isquemia caliente se inicia desde la oclusión de la arteria renal hasta la inmersión del riñón en hielo ${ }^{9}$. La función retardada de injerto es la necesidad de diálisis en la primera semana de trasplante $^{9}$.

Los datos fueron recolectados en protocolos diseñados y los resultados fueron expresados en porcentajes.

\section{RESULTADOS}

Se realizó un estudio prospectivo y descriptivo de las nefrectomías derechas de donantes vivo por laparoscopia seleccionados para este procedimiento desde mayo 2006 hasta septiembre 2008 en la Unidad de Trasplante del Hospital Militar Dr. Carlos Arvelo, en la cual fueron 10 donantes. Se excluyó 1 paciente debido a lesión retroperitoneal por la aguja de Veress por lo que ameritó la conversión a técnica abierta.

Todos los donantes fueron del sexo femenino, con una edad promedio de 30,7 años (20-37 años). $\mathrm{El}$ índice de masa corporal promedio fue de 24,4 $\mathrm{Kg} / \mathrm{m}^{2}\left(17,90-32,5 \mathrm{Kg} / \mathrm{m}^{2}\right)$ (Tabla 1$)$.

$\mathrm{El}$ criterio de lateralidad que predominó en todos los casos fue que eran donantes femeninas en edades reproductivas. En 8 pacientes el volumen renal derecho era menor comparado con el izquierdo, con una diferencia mayor de 5 cc. En un caso presentó una anatomía vascular compleja izquierda.

$\mathrm{El}$ abordaje en todos los casos fue transperitoneal. El tiempo quirúrgico promedio fue de 158.3 minutos (110-270 minutos). La pérdida de sangre promedio fue de $272 \mathrm{cc}$ (50-700 cc), en un solo caso ameritó transfusión de sangre. La isquemia caliente promedio fue de 3,2 minutos (2-4 minutos).

La cirugía reconstructiva con prótesis vascular Gorotex se realizó en 6 injertos renales, debido a la longitud corta de la vena renal y 3 de ellos presentaban doble vena renal (ver tabla 1).

El inicio de la vía oral fue entre las 6 a 24 horas, con una estancia hospitalaria promedio de 1.6 días.

Los donantes no presentaron complicaciones, mientras que un receptor presentó función retardada de injerto $(12,5 \%)$ (Tabla 1). Ninguno presento trombosis venosa.

\section{DISCUSIÓN}

La necesidad de aumentar la oferta de órganos para así lograr el trasplante renal ha llevado a pensar en el donante vivo, que en sus inicios el abordaje fue 
Tabla 1. Datos de los donantes renales

\begin{tabular}{|c|c|c|c|c|c|c|c|c|}
\hline Sexo & IM & $\begin{array}{c}\text { Arteria/ } \\
\text { Vena }\end{array}$ & $\begin{array}{c}\text { Tiempo } \\
\text { (min) }\end{array}$ & $\begin{array}{c}\text { Sangrado } \\
\text { (cc) }\end{array}$ & $\begin{array}{c}\text { Isquemia } \\
\text { caliente (seg) }\end{array}$ & Banco & $\begin{array}{c}\text { Estancia } \\
\text { hospitalaria }\end{array}$ & Complicaciones \\
\hline Femenina & 24,9 & $1 / 2$ & 150 & 100 & 200 & Sí & 2 & No \\
\hline Femenina & 17,9 & $1 / 1$ & 150 & 50 & 180 & No & 1 & No \\
\hline Femenina & 19,1 & $1 / 2$ & 270 & 400 & 180 & Sí & 2 & No \\
\hline Femenina & 20 & $1 / 2$ & 200 & 100 & 270 & Sí & 2 & No \\
\hline Femenina & 29,2 & $1 / 1$ & 120 & 200 & 240 & No & 1 & No \\
\hline Femenina & 29,5 & $1 / 1$ & 135 & 200 & 180 & Sí & 1 & No \\
\hline Femenina & 32,4 & $1 / 1$ & 110 & 700 & 120 & Sí & 2 & No \\
\hline Femenina & 26,3 & $1 / 1$ & 160 & 400 & 240 & Sí & 2 & No \\
\hline Femenina & 20,4 & $1 / 1$ & 130 & 300 & 180 & No & 2 & DRI \\
\hline
\end{tabular}

DRI: Función retardada de injerto

por técnica abierta. Con la descripción de Ratner ${ }^{4}$ )de la técnica de la nefrectomía de donante por laparoscopia en 1.995 generó un gran interés debido a las bondades de la cirugía mínimamente invasiva, como son menos dolor, retorno al trabajo más rápido y mejor resultado cosmético. Actualmente el abordaje laparoscópico es la técnica estándar en muchos centros de trasplante ${ }^{7}$.

En la selección del riñón a extraer es importante mantener el mejor riñón in situ con el donante ${ }^{10}$. Históricamente, el porcentaje de nefrectomías derechas en cirugía abierta oscila entre 26 a $37 \%^{11}$, mientras que el abordaje laparoscópico se ubica entre 2,5 a $15 \% 0^{5,12}$.

Las indicaciones absolutas para la selección del lado derecho son: riñón izquierdo mas grande, presencia de quiste renales y displasia fibromuscular leve derecha; mientras que las relativas tenemos: anatomía vascular compleja o aberrante del lado izquierdo, doble sistema colector o ptosis renal derecha $^{7}$. En el caso de mujeres en edad fértil con igual función renal, el riñón derecho debe ser escogido, debido a que la pielonefritis e hidronefrosis durante la gestación es más común en este lado ${ }^{13}$. Bettschat $^{14}$ añade como criterio de lateralidad la función renal a través de la realización de gammagrama renal MAG3, observando que 9 de los 13 riñones derechos fueron por mejor función del riñón izquierdo. En nuestra casuística encontramos que 8 de los 9 donantes el criterio de selección fue la diferencia de volumen renal, todas las donantes fueron del sexo femenino en edades reproductivas como criterio de selección del lado derecho, y en un solo caso la anatomía vascular izquierda era compleja.
Aunque la laparoscopia es el abordaje estándar en la nefrectomía de donante ${ }^{7}$, esta técnica en un principio fue limitada al riñón izquierdo debido a que la vena renal es más larga y la exposición de la arteria renal es menos compleja. Mandal ${ }^{6}$ reporta inicialmente en la nefrectomía derecha de donante vivo $37.5 \%$ de trombosis venosa atribuido a la longitud y lo delgado de la pared de la vena renal, observando que el uso de la engrapadora endovascular GIA (United States surgical, Norwalk, CT) resulta en una perdida obligatoria del vaso que oscila de 1 a 1,5 $\mathrm{cm}^{6,8}$ agravando la longitud corta de la vena renal derecha. A partir de esto, Mandal ${ }^{6}$ plantea 3 modificaciones para disminuir el impacto de esta reducción de longitud de la vena renal, que son: colocar la engrapadora endovascular paralela a la vena cava inferior, realizar una incisión de 5 a 6 cm subcostal para el uso de clamp de Satinsky estándar o el uso de injerto de vena safena del receptor para obtener mayor longitud de la vena renal. Otros autores plantean el uso de clamp vascular Satinsky atraumático (Aesculap, Inc., Center Valley, Pennsylvania) a fin de duplicar la cirugía abierta y obtener un parche de vena cava inferior ${ }^{15}$, la técnica de mano asistida en la cual facilita la extensión de la vena renal y que las líneas de grapas incorpore una pequeña porción de la vena cava inferior $^{8}$, la engrapadora Endo-TA (United States Surgical Corp., Norwalk, Connecticut) en la cual coloca 3 líneas de grapas sin división de la vena renal $^{8,12}$, el abordaje retroperitoneal visualizando el espacio inter-aortocava por lo que asegura una mayor longitud de la vena renal ${ }^{16}$ y el Hem-o-lock ${ }^{\circledR}$ (Weck Closure Systems, Research Triangle Park, 
NC, EUA) ${ }^{17}$. En nuestra casuística usamos 2 Hemo-lock $^{\circledR}$ (Weck Closure Systems, Research Triangle Park, NC, EUA) en la vena renal ocasionado una pérdida de longitud de 4 a $5 \mathrm{~mm}$, siendo imperativo que los vasos renales estén libres de tejido adiposo y linfático de manera de evitar la dislocación del Hem-o-lock ${ }^{\circledR}$.

Otros reportes de incidencia de trombosis venosa en riñón derecho se ubican entre el 0 y 4\% $\%^{8,12,18}$. En nuestra serie no reportamos ninguna trombosis venosa. Es importante realizar una anastomosis venosa libre de tensión, para esto la disección de la vena iliaca externa debe ser completa ${ }^{6}$, y si fuese necesario la división de la venas lumbares y de la ilíaca interna ${ }^{18}$, además de prolongar la vena renal con safena del receptor ${ }^{6}$. En nuestro estudio, se utilizo prótesis vasculares con Gorotex en 6 injertos renales, 3 de ellos tenias doble vena renal, sin tener complicaciones vasculares en el receptor.

El tiempo quirúrgico de la nefrectomía derecha de donante por laparoscopia es menor comparada con el lado izquierdo ${ }^{19,20}$, ya que obvia la división de la vena gonadal, lumbar y adrenal. En nuestro estudio encontramos que este tiempo fue de 158,3 minutos en cual es similar a otros reportes ${ }^{19,20}$. De igual manera, el tiempo de isquemia caliente es comparable a otras series ${ }^{18}$.

En nuestra casuística encontramos un solo caso de función retardada de injerto $(12,5 \%)$, menor a la reportada por Lind que fue de $23 \%$ para la nefrectomía derecha ${ }^{19}$.

\section{CONCLUSIÓN}

La nefrectomía derecha de donante vivo por laparoscopia ofrece una excelente calidad de injerto. El desafío de una longitud venosa corta puede ser resuelta con la liberación adecuada de la vena ilíaca o el uso de próstesis vasculares sin presentar complicaciones. Nosotros creemos que la laparoscopia en la nefrectomía derecha de donante es técnicamente factible, segura y equivalente a la cirugía abierta, permitiendo establecer los mismos criterios de selección que en la cirugía abierta y manteniendo el principio de dejar el mejor riñón al donante.

\section{REFERENCIAS}

1. Novotny M. Laparoscopic live donor nephrectomy. Urol Clin North Am. 2001 Feb;28(1):127-135.

2. Wolfe RA, Ashby VB, Milford EL, Ojo AO, Ettenger RE, Agodoa LY, Held PJ, Port FK. Comparison of mortality in all patients on dialysis, patients on dialysis awaiting transplantation, and recipients of a first cadaveric transplant. N Engl J Med. 1999 Dec 2;341(23):1725-1730.
3. Gill IS, Carbone JM, Clayman RV, Fadden PA, Stones MA, Lucas BA, McRoberts JW. Laparoscopic live donor nephrectomy. J Endourol. 1994;8(2):143-148.

4. Ratner LE, Ciseck LJ, Moore RG, Cigarroa FG, Kaufman HS and Kavoussi LR. Laparoscopic live donor nephrectomy. Transplantation 1.995;60: 1047-1049.

5. Jacobs SC, Cho E, Dunkin BJ, Flowers JL, Schweitzer E, Cangro C, Fink J, Farney A, Philosophe B, Jarrell B, Bartlett ST. Laparoscopic live donor nephrectomy: the University of Meryland 3-year experience. J Urol 2.000; 164: 1494-1499.

6. Mandal AK, Cohen C, Montgomery RA, Kavoussi LR, Ratner LE. Should the indications for laparoscopic live donor nephrectomy of the right kidney be the same as for the open procedure? Anomalous left renal vasculature left donor nephrectomy. Transplantation. 2001 Mar 15;71(5):660-664.

7. Del Pizzo J. Laparoscopic live donor nephrectomy: Technique. Gill I. Editor. Textbook of laparoscopic urology. New York. Informa Healthcare Inc. 2.006, pp 337-352.

8. Buell JF, Hanaway MJ, Potter SR, Koffron A, Kuo PC, Leventhal J, Cho E, Johnson M, Edye M, Woodle ES.. Surgical techniques in right laparoscopic donor nephrectomy. J Am Coll Surg. 2002;195(1):131-137.

9. Jacobs SC, Cho E, Foster C, Liao P, Bartlett ST. Laparoscopic donor nephrectomy: The University of Maryland 6-year experience. J Urol 2004; 171(1):47-51.

10. Murray JE. and Harrison JH. Surgical management of fifty patients with kidney transplants including eighteen pairs of twins. Am J Surg. 1963;105:205-18.

11. Barry JM. Editorial: Living donor nephrectomy. J Urol 2004; 171:6162.

12. Abrahams HM, Freise CE, Kang SM, Stoller ML, Meng MV.. Technique, indications and outcomes of pure laparoscopic right donor nephrectomy. J Urol 2004; 171: 1793-1796.

13. Stevens W. Comment on dilation of the kidney pelvis and ureter during pregnancyand the puerperium: a pyelographic study in normal women. JAMA 1933;101;2029-2031.

14. Bettschart V., Boubaker A., Martinet O., Golshayan D., Wauters J. and Mosimann F. Laparoscopic right nephrectomy for live kidney donation: functional results. Transpl Int. 2003;16(6):419-424.

15. Turk IA, Deger S, Davis JW, Giesing M, Fabrizio MD, Schönberger B, Jordan GH, Loening SA. Laparoscopic live donor right nephrectomy: a new technique with preservation of vascular length. $J$ Urol. 2002;167(2 Pt 1):630-633.

16. Gill IS, Uzzo RG, Hobart MG, Streem SB, Goldfarb DA, Noble MJ. Laparoscopic retroperitoneal live donor right nephrectomy for purposes of allotransplantation and autotransplantation. J Urol. 2000;164 (5):1500-1504.

17. Mitre A, Dénes F, Simoes F, Videolaparoscopia para doador renal vivo: técnica. Batista M, Castro S, Fonseca G and Franco F. Editors. Sao Paulo. Roca. 2007, pp 159-166

18. Lind MY, Hazebroek EJ, Hop WC, Weimar W, Jaap Bonjer H, IJzermans JN. Right side laparoscopic live donor nephrectomy: is reluctance still justified? Transplantation. 2002 Oct 15;74(7):1045-1048.

19. Buell JF, Edye M, Johnson M, Li C, Koffron A, Cho E, Kuo P, Johnson L, Hanaway M, Potter SR, Bruce DS, Cronin DC, Newell KA, Leventha J, Jacobs S, Woodle ES, Bartlett ST, Flowers JL. Are concerns over right laparoscopic donor nephrectomy unwarranted? Ann Surg. 2001; 233(5):645-51.

20. Posselt AM, Mahanty H, Kang SM, Stoller ML, Meng MV, Roberts JP, Freise CE. Laparoscopic right dono nephrectomy: a large single center experience. Transplantation 2004;78(11):1665-1669.

Correspondencia autor: Dr. R.J. de Andrade Andrade

Hospital Militar Dr. Carlos Arvelo

Avda. José Angel Lamas. 1020 Caracas (Distrito Federal)

Venezuela. Tel.: 58-212-4061685

E-mail autor: rdeandrade@cantv.net

Información Artículo:

Trabajo recibido: octubre 2008

Trabajo aceptado: noviembre 2008 